\title{
Srf KO and wild-type mice similarly adapt to endurance exercise
}

\author{
Haidar Djemai* (1,2), Medhi Hassani* (3,4,5), Nissrine Daou (3), Zhenlin Li (3), \\ Athanassia Sotiropoulos (6), Philippe Noirez $(1,2,7)$, Dario Coletti $(3,4,5)$
}

(1) Université Paris Descartes, Sorbonne Paris Cité, Paris, France; (2) IRMES, INSEP, Paris, France; (3) Sorbonne University, Paris, France; (4) Sapienza University of Rome, Rome, Italy; (5) Interuniversity Institute of Myology, Rome, Italy; (6) Institut National de la Santé et de la Recherche Médicale U1016, Institut Cochin, Paris, France; (7) Department of Exercise Science, UQAM, Montréal, Canada. * = equal contribution

This article is distributed under the terms of the Creative Commons Attribution Noncommercial License (CC BY-NC 4.0) which permits any noncommercial use, distribution, and reproduction in any medium, provided the original author(s) and source are credited.

\begin{abstract}
Physical exercise has important effects as secondary prevention or intervention against several diseases. Endurance exercise induces local and global effects, resulting in skeletal muscle adaptations to aerobic activity and contributes to an amelioration of muscle performance. Furthermore, it prevents muscle loss. Serum response factor (Srf) is a transcription factor of pivotal importance for muscle tissues and animal models of Srf genetic deletion/over-expression are widely used to study Srf role in muscle homeostasis, physiology and pathology. A global characterisation of exercise adaptation in the absence of Srf has not been reported. We measured body composition, muscle force, running speed, energy expenditure and metabolism in WT and inducible skeletal muscle-specific Srf KO mice, following three weeks of voluntary exercise by wheel running. We found a major improvement in the aerobic capacity and muscle function in WT mice following exercise, as expected, and no major differences were observed in Srf KO mice as compared to WT mice, following exercise. Taken together, these observations suggest that Srf is not required for an early (within 3 weeks) adaptation to spontaneous exercise and that Srf KO mice behave similarly to the WT in terms of spontaneous physical activity and the resulting adaptive responses. Therefore, Srf KO mice can be used in functional muscle studies, without the results being affected by the lack of Srf. Since lack of Srf induces premature sarcopenia, our observations suggest that the modifications due to the absence of Srf take time to occur and that young, Srf KO mice behave similarly to WT in aerobic physical activities.
\end{abstract}

Key Words: Exercise training, Serum response factor, $\dot{\mathrm{VO}}_{2}$, RER, Performance.

Eur J Transl Myol 29 (2): 97-105, 2019

Resistance and endurance exercises induce structural and functional adaptations in skeletal muscle. ${ }^{1-3}$ Adaptations to endurance exercise include an increase in muscle fiber mitochondrial content and respiratory capacity; a slower utilization of muscle glycogen and blood glucose, a greater reliance on fat oxidation, and a lower lactate production. ${ }^{4}$ Due to the aforementioned, beneficial effects of exercise, the latter has been widely used in animal experimental models. ${ }^{5-9}$ The most classical training prescription method for aerobic training is based on tests using calorimetric parameters that determine the physical activity performance, such as maximal oxygen consumption $\left(\dot{\mathrm{V}}_{2 \max }\right)$, which represents the highest rate at which oxygen is extracted in the lungs, transported, and used by the body during maximal exercise, and maximal running speed (Smax), defined as the maximum velocity reached during an incremental test. Srf is a transcription factor highly expressed in skeletal, cardiac and smooth muscles, playing a key role in regulating skeletal muscle mass, function and homeostasis. ${ }^{10,11}$ Srf is a ubiquitously expressed member of the MADS box family, which binds the core sequence of CArG boxes [CC (A/T) $6 \mathrm{GG}] .{ }^{12}$ Among Srf transcriptional targets some are specifically expressed in skeletal muscle, including alpha actins, myosin light chain, dystrophin, muscle creatine kinase, myoD and several genes encoding sarcomeric proteins. ${ }^{13}$ Rho family of small GTPases and actin dynamics have been shown to control the nuclear accumulation of Srf coactivator Mrtf-A and therefore modulate Srf activity. ${ }^{14,15}$ Data obtained from mouse genetic models with skeletal muscle specific loss of Srf or Mrtfs 
functions emphasize their crucial role in post-natal muscle growth. ${ }^{16-19}$ In adults, Srf activity controls skeletal muscle mass. Indeed, Srf loss blunts overloadinduced hypertrophy and worsens disuse atrophy. ${ }^{20,21-25}$ Srf transcriptional activity is up-regulated by biomechanical stimuli via integrin signaling. ${ }^{22}$ Srf also affects vascular muscular tone and arterial stiffness, ${ }^{23}$ and lack of Srf induces dilated cardiomyopathy. ${ }^{24}$ In all these cases mechanical stimulation plays an important role. Based on the scientific literature indications, highlighting the role played by Srf in skeletal muscle adaptation to exercise is of great interest. Therefore, we exploited a mouse model of targeted, conditional and inducible invalidation of Srf using the Cre-LoxP system by crossing mice homozygous for Srf floxed alleles (Sf/Sf) with HSA-Cre-ERT2 transgenic mice ${ }^{18,26}$ which express a tamoxifen (TAM) dependent Cre recombinase exclusively in post-mitotic myofibers. With the aim to investigate the effects of endurance exercise on Srf knockout (KO) mice adaptation to exercise, we investigated the effect of voluntary exercise on body weight, muscle mass and strength. Here, we show that Srf KO mice adapt to endurance exercise like wild-type mice. Thus, Srf KO mice can be used as an animal model for studies involving endurance exercise protocols, without the risks of bias due to the lack of Srf expression.

\section{Materials and Methods}

\section{Animals}

In total 16 female mice aged 8 weeks were used for the experiments, including 8 knock-out (KO) mice for the Srf gene and 8 wild-type (WT) mice. These mice had a mixed genetic background (second generation of backcross between Balb/c AnRj and C57BL/6 strains). The mice were fed ad libitum during the protocol period and were exposed to day / night cycles of $12 \mathrm{~h} / 12 \mathrm{~h}$. The study was approved by the Paris Descartes Animal Experiment Committee (Project No. 2017051916196640) and performed according to the procedures of the Guide to the Care and Use of Laboratory Animals published by NIH (No. 85-23, revised 1996) and approved by the French Ministry of Agriculture. Mice homozygous for Srf floxed alleles 27 (abbreviated to Sf/Sf) and HSA-CreERT2 transgenic mice have been described elsewhere. ${ }^{26}$ These two mouse strains were backcrossed onto a C57BL/6N genetic background for 7 generations prior to experimental analysis and then crossed to generate double transgenic mice HSA-Cre-ERT2:Sf/+. Subsequent breeding of HSA-Cre-ERT2:Sf/+ and Sf/Sf mice generated HSA-Cre-ERT2:Sf/Sf pre-mutant mice. Mice were genotyped by PCR, using DNA extracted from tail biopsies. In all experiments, groups of 2 months

A)

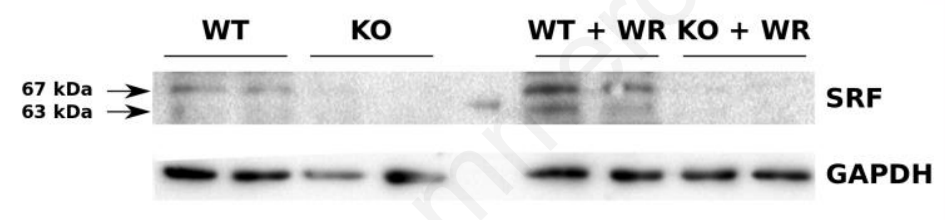

B)

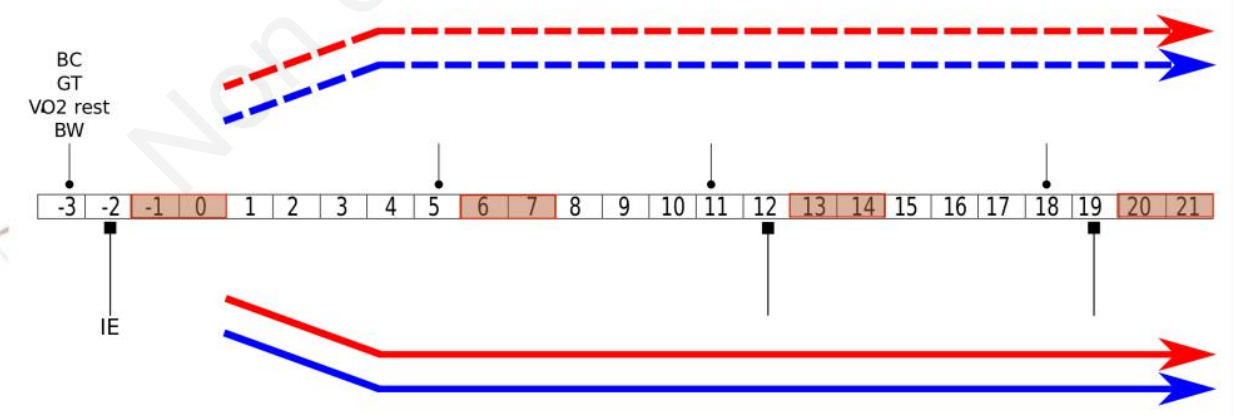

Fig 1. Protocol design and validation of the skeletal muscle-specific SRF KO model after TAM injection.

A) Immunoblot analysis of protein extracts from wild-type and Srf KO muscles of control and trained mice after TAM injection using an anti-SRF antibody. Anti-GAPDH is used as a loading control. B) Flowchart representing the experimental design with the different protocols and the duration of the study. The age of the mice at the beginning of the study was 8 weeks. The control group (C), composed of 4 wild-type (WT) mice (blue dashed line type) and 4 knockout $(K O)$ mice for the Srf gene (red dashed line type), does not have access to a running wheel. The trained group $(E)$ composed of 4 WT mice (blue continuous line type) and $4 \mathrm{KO}$ (red continuous line type), follows a 3-week wheel running training protocol. Body weight, body composition measurements, gript test and VO2 rest (round head arrow) and the physical performance assessments (square head arrow) were performed as indicated in the flowchart. 
Table 1. Exercise-dependent changes in body weight and body composition

\begin{tabular}{|c|c|c|c|c|c|c|c|c|}
\hline & & & \multicolumn{2}{|c|}{ Control group (C) } & \multicolumn{2}{|c|}{ Trained group (E) } & \multirow{4}{*}{$\begin{array}{c}\text { Trained } \\
\text { effect }\end{array}$} & \multirow{4}{*}{$\begin{array}{c}\text { Genotype } \\
\text { effect }\end{array}$} \\
\hline & WT $(n=8)$ & $\mathrm{KO}(\mathrm{n}=8)$ & & & & & & \\
\hline & \multirow[t]{2}{*}{ T0 } & T0 & & & & & & \\
\hline & & & WT $(n=4)$ & $\mathrm{KO}(\mathrm{n}=4)$ & WT $(n=4)$ & $\mathrm{KO}(\mathrm{n}=4)$ & & \\
\hline Body weight (g) & $23.75 \pm 2.75$ & $23.60 \pm 3.10$ & $23.38 \pm 1.73$ & $23.83 \pm 2.66$ & $24.25 \pm 2.11$ & $24.05 \pm 2.40$ & NS & NS \\
\hline Lean mass $(\mathrm{g})$ & $12.70 \pm 1.17$ & $12.77 \pm 1.23$ & $13.29 \pm 0.89 * * *$ & $13.55 \pm 1.73^{*}$ & $13.71 \pm 1.00$ & $13.92 \pm 1.33 * *$ & NS & NS \\
\hline Lean mass/Body weight (\%) & $0.54 \pm 0.03$ & $0.54 \pm 0.03$ & $0.57 \pm 0.02$ & $0.57 \pm 0.02$ & $0.57 \pm 0.02 *$ & $0.58 \pm 0.01^{* *}$ & NS & NS \\
\hline Fat mass $(\mathrm{g})$ & $2.79 \pm 1.42$ & $2.57 \pm 1.25$ & $2.12 \pm 0.29$ & $1.90 \pm 0.76$ & $2.22 \pm 0.94$ & $1.95 \pm 0.85$ & NS & NS \\
\hline Fat mass/Body weight (\%) & $0.11 \pm 0.05$ & $0.11 \pm 0.04$ & $0.09 \pm 0.01$ & $0.08 \pm 0.03$ & $0.09 \pm 0.04$ & $0.08 \pm 0.03$ & NS & NS \\
\hline
\end{tabular}

Wild-type (WT) and Srf KO mice were analysed 3 days before (T0) and 3 weeks after (T3) the beginning of the training. Body composition was measured by nuclear magnetic resonance system. Mean $\pm \mathrm{SD}$. Difference between T0 vs. T3: ${ }^{*} p<0.05,{ }^{* *} p<0.01,{ }^{* * *} p<0.001$. (With linear mixed-models approach with repeated measures ANOVA and simultaneous tests for general linear hypotheses). Training effect: difference between $\mathrm{C} v \boldsymbol{s}$. E. Genotype effect: WT vs. KO.

old male pre-mutant (HSA-Cre-ERT2:Sf/Sf) and sexand aged matched control (Sf/Sf) mice were given daily intraperitoneal TAM (1 mg per day; Sigma) injections for 5 consecutive days. Cre-mediated excision of Srf floxed

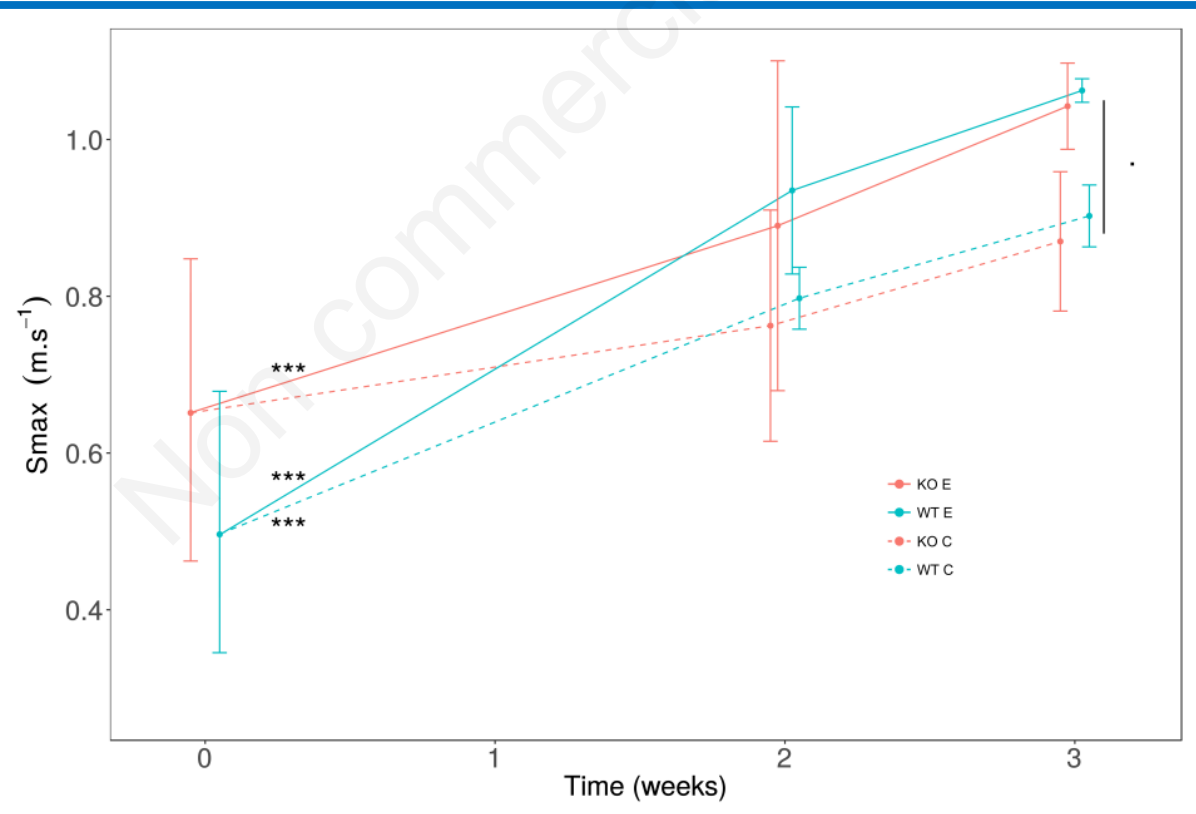

Fig 2. Effect of voluntary activity on maximal speed in Srf gene knockout mice

Incremental speed tests were carried out at the beginning (0), after 2 weeks and after 3 weeks of training. Following a warm-up period of $0.05 \mathrm{~cm} . \mathrm{s}^{-1}$, the speed of the treadmill is incremented by $1 \mathrm{~cm} . \mathrm{s}^{-1}$ every $15 \mathrm{sec}$. Smax is the maximum speed reached by each mouse corresponding to the end of the test. Wild-type (WT in blue; $n=8$ ) and Srf gene knockout (KO in red; $n=8)$, 8-week-old female mice were divided in 2 groups: trained (E, continuous line type) and control $(C$, dashed line type). WT $(E)$ and $K O(E)$ both significantly increased their Smax after 3 weeks of training in respect to the TO. WT $(C)$ had a significant increase in Smax at TO even in the absence of training. With a global analysis $E$ vs $C$ at week 3 , we found a trend of improvement for Smax in $(E)$ with a $p=0.08$. Mean $(d o t) \pm S D$. Differences between T0 vs. T3: *** $p<0.001$. Difference between $C$ vs. $E: p=0.08$. 


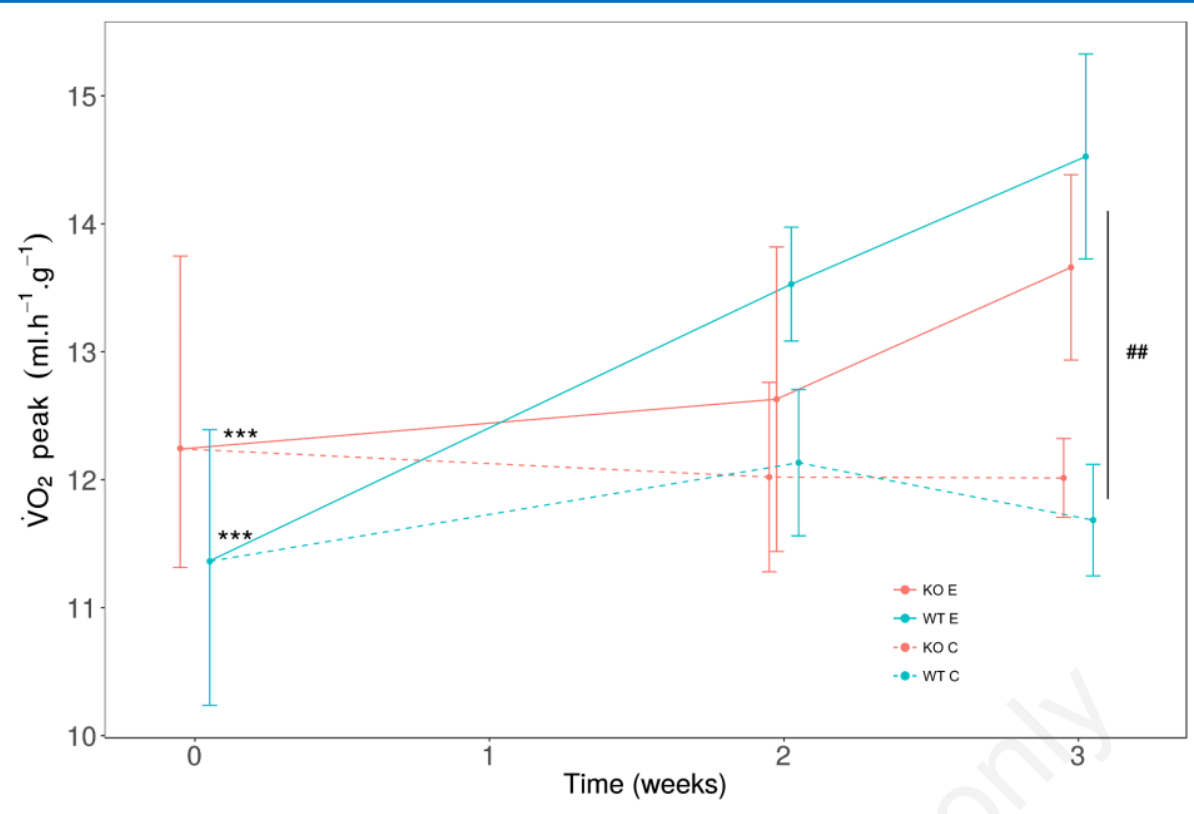

Fig 3. Effect of voluntary activity on oxygen consumption $\left(\dot{V} \mathrm{O}_{2}\right)$ peak in Srf KO mice.

The oxygen consumption (VO2) was measured at the beginning (0), after 2 weeks and after 3 weeks of training. The $\dot{V} \mathrm{O}_{2}$ peak was measured by an incremental speed test on individual treadmill equipped with a calorimetric system. The speed was increased by $1 \mathrm{~cm} . \mathrm{s}^{-1}$ every $15 \mathrm{sec} . \dot{V} \mathrm{O}_{2}$ was expressed relatively to lean mass ( $\mathrm{ml} . \mathrm{h}^{-1} \cdot \mathrm{g}^{-1}$ ). Wild-type (WT in blue) $(n=8)$ and Srf gene knockout (KO in red) (n=8) 8-week-old female mice were divided in 2 groups: trained (E, continuous line type) and control $\left(C\right.$, dashed line type). The trained group (E) significantly increase $\dot{V} O_{2}$ peak in 3 weeks, for both $W T$ and $K O$ genotypes, but not the control group $(C)$. At 3 weeks, we observed a significant difference between $(C)$ and (E). Mean $(d o t) \pm S D$. Differences between T0 vs. T3: ***P<0.001. Difference between $C$ vs. E: \#\# P=0.01.

alleles was detected as previously described. ${ }^{27}$ Efficiency of the deletion was validated by WB (figure 1A).

\section{Western blot analysis}

TA muscles were dissected, cut and homogenized in 0.3 $\mathrm{ml}$ of lysis buffer (50mM Tris-HCl pH 7.4, 1mM EDTA, $150 \mathrm{mM} \mathrm{NaCl}, 1 \%$ TRITON) supplemented with protease and phosphate inhibitors (complete Mini EDTA free and PhosSTOP, Roche). $30 \mathrm{mg}$ of proteins were separated by means of SDS-PAGE with $12 \%$ of acrylamide and transferred to PVDF membrane (Invitrogen). Unspecific bindings were blocked in 5\% non-fat dry milk (Santa Cruz) in TBST $1 \times(20 \mathrm{mM}$ Tris, $137 \mathrm{mM} \mathrm{NaCl}, 0.1 \%$ Tween 20) for 1 hour at room temperature; membranes were then incubated at $4{ }^{\circ} \mathrm{C}$, overnight, with primary antibody diluted in $4 \%$ milk (Sigma) in TBST. The SRF (Santa Cruz) and GAPDH (Santa Cruz) antibodies, diluted to 1:1000 and 1:10000, respectively, were used. After washing in TBST, membranes were incubated for 1 hour at room temperature with HRP-conjugated antimouse or anti-rabbit secondary antibodies (Biorad) diluted 1:10000 in TBST, and signals were detected using ECL reagent (Cyanagen). Intensity of Western blot signals was quantified by using ImageLab software.

Protocol design and voluntary exercise

Both KO and WT mice were divided into two groups of four mice each for every experimental set: (1) control or sedentary mice (C); (2) mice subjected to voluntary training (E). The latter were installed in individual cages equipped with a wheel (BIO-ACTIVW - Bioseb, France) connected to a digital counter of wheel revolutions and time of the exercise (Figure $1 \mathrm{~B}$ ). Data were recorded daily for a period of 3 weeks. ${ }^{28}$ The evaluation of the physical performance of the mice took place at 4 moments: just before the beginning of the protocol (T0) and 1, 2 and 3 weeks after the beginning of the training protocol (T1, T2, and T3, respectively) (Figure $1 \mathrm{~B}$ ). These evaluations were carried out in the facility "Performance \& Metabolism in Mice" (PMM, EA7329, Paris Descartes University).

\section{Body composition}

The body weight of the mice was registered daily, five times per week. Their body composition, such as lean body mass and body fat, were measured weekly at T0, T1 $\mathrm{T} 2$ and $\mathrm{T} 3$ by a nuclear magnetic resonance system (LF90II, Bruker, Germany).

\section{Evaluation of gripping strength}

To evaluate the grip strength, we used the Strength Meter Grip (BIO-GS3, Bioseb, France). The force measurements were performed weekly at T0, T1, T2 and $\mathrm{T} 3$. The test was repeated 5 times in a row with a recovery time of 30 seconds between each test. The average value (Fmean) as well as the maximum value (Fmax) of the 5 


\section{Srf KO and WT mice adaptation to endurance exercise}

Eur J Transl Myol 29 (2): 97-105, 2019

tests were recorded as the gripping force of the forelimb and hindlimb for each mouse.

\section{Incremental exercise on a treadmill}

For the evaluation of the physical performance we used a single-track unidirectional treadmill with a speed adjustable from 0 to $1.07 \mathrm{~m} . \mathrm{s}^{-1}$, equipped with a calorimetric cage (Phenomaster, TSE, Germany). The mice had an acclimation period of $10 \mathrm{~min}$ at a speed of $0.05 \mathrm{~m} . \mathrm{s}^{-1}, 24$ hours before the beginning of the protocol. The running speed had not been increased further, to avoid a training effect. The mice were acclimated on the treadmill 3 min just before evaluation at low rates of 0.01 to $0.05 \mathrm{~m} . \mathrm{s}^{-1}$. In order to evaluate the physical performance of the mice, the mice were exercised at an incremental speed, which was increased by $1 \mathrm{~cm} . \mathrm{s}^{-1}$ every 15 seconds. Oxygen consumption $\left(\dot{\mathrm{V}}_{2}\right)$, carbon dioxide production $\left(\dot{\mathrm{V} C \mathrm{CO}_{2}}\right)$ and respiratory exchange ratio (RER $\left.=\dot{\mathrm{V} C O} \mathrm{O}_{2} / \dot{\mathrm{VO}}_{2}\right)$ were recorded every second. The peak oxygen consumption $\left(\dot{\mathrm{VO}}_{2}\right.$ peak in $\left.\mathrm{ml}^{-1} \mathrm{~h}^{-1}\right)$ and $\mathrm{RER}_{\text {peak }}$ were defined as the highest values achieved during exercise. ${ }^{29-33}$ Stopping the exercise corresponds to the maximum speed (Smax in $\mathrm{m} . \mathrm{s}^{-1}$ ) reached by each mouse.

\section{Indirect calorimetry at rest}

The resting $\dot{\mathrm{VO}}_{2}$ and RER were measured for each mouse on the stationary treadmill equipped with the calorimetric cage. The $\dot{\mathrm{VO}}_{2}$ rest and $\mathrm{RER}_{\text {rest }}$ correspond to the lowest values over a period of 1 hour.

\section{Statistical analyses}

Data were expressed as mean \pm standard deviation (SD). All statistical analyzes were performed using the software R (3.4.4) (R Foundation for Statistical Computing, Vienna, Austria). After assessing variance homogeneity with levene test, statistical analyzes were performed using the linear mixed-models approach (nlme package) with repeated measures ANOVA to evaluate interactions between the training ( $\mathrm{C}$ vs $\mathrm{E}$ ) and the genotype (WT vs KO) effect during the protocol (T0, T1, T2 and/or T3). Post-hoc analyses were conducted with simultaneous tests for general linear hypotheses (emmeans package). The statistical significance threshold was set at $\mathrm{p}<0.05$.

\section{Results}

\section{Exercise increases lean mass in WT and Srf KO mice}

Since physical activity affects body mass and composition, we measured the changes in both BW and body composition in mice in the absence or presence of exercise training, in both WT and Srf KO mice (Table 1). Body weight showed no significant difference between sedentary and trained mice regardless of Srf expression. Accordingly, the body composition (lean mass and fat mass) showed no major differences between group $\mathrm{C}$ and $\mathrm{E}$; even though we could measure an increase of lean body mass in the control groups (C) and not in the exercised groups (E), independently of Srf expression, we cannot exclude that the latter, negative result is a false negative (Table 1). When normalizing lean or fat mass by body weight, we noticed that lean mass increased specifically in the exercised groups (E), as expected, with no significant differences between WT and Srf KO mice (Table 1). It is worthwhile to note that fat mass was not affected by exercise in both conditions.

\section{Exercise effects on strength are amplified in Srf $\mathrm{KO}$ mice}

The observed increase in lean mass, i.e. muscle mass, prompted us to investigate whether exercise had any effect on muscle function and whether Srf expression affected muscle response. As a functional assay we performed the non-invasive, repeatable grip test and we recorded two different parameters: the mean strength (the average of repeated measures on the same animal) and the maximal strength (the highest value observed in the repeated measures). In control conditions ( $C$ groups) we noticed a significant increase in the strength of the WT mice over time, which was absent in the Srf KO mice, initially suggesting that their muscle performance could be negatively affected by the lack of Srf (Table 2). However, upon exercise the Srf KO mice showed a significant amelioration of the muscle strength, which appeared to be even higher than WT mice (the latter showed just a positive trend, Table 1). Upon normalization by lean mass, the average force increased significantly only in trained $\mathrm{KO}$ mice (E groups in Table 2 ), suggesting that Srf KO mice not only do not have a deficit in specific force as compared to WT but show indeed an even greater response to exercise training.

Voluntary exercise ameliorates the running performance and endurance of both WT and Srf KO mice

Endurance exercise is known to induce muscle adaptations leading to increased physical performance in a few weeks in mice, thus, we analyzed the effects of voluntary exercise in terms of maximal running speed (Smax, Figure 2). We noticed that the training induced a significant increase in Smax, both in WT and Srf KO mice, suggesting that exercise did not exert differential effects depending on the genotype (Figure 2). Individual comparisons pinpointed a significant increase in the maximal speed at T3 versus T0 in WT mice, both in the absence and in the presence of exercise; and, more important, exercised Srf KO mice also showed an improvement in the maximal speed at T3, indicating that they were capable to benefit of the endurance effects of the training period similarly to the WT counterpart (Figure 2, Table 2). The endurance capacity of the mice was measured by the $\dot{\mathrm{V}} \mathrm{O}_{2 \text { peak, }}$ since this parameter suggests a higher aerobic capacity. The trained mice showed a significant increase of $\mathrm{VO}_{2}$ peak between $\mathrm{T} 0$ and T3, while the control group had not changed in the same period; in both cases, this phenomenon occurred independently of the genotype (Figure 3, Table 2). To shed light on the metabolic adaptations associated to 


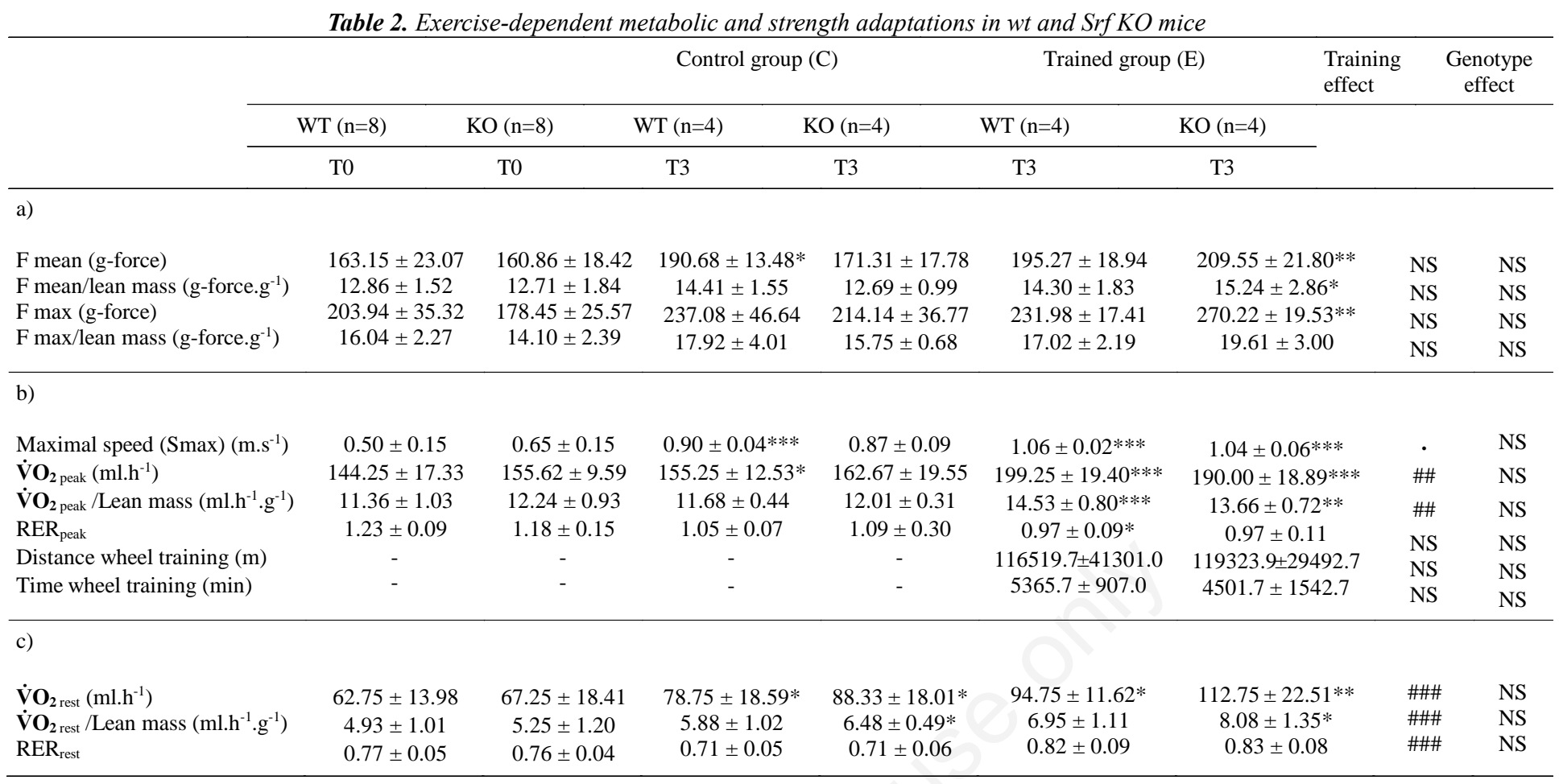

Wild-type (WT) and KO mice were analysed 3 days before (T0) and 3 weeks after (T3) the beginning of the training: a) strength was measured by grip test, b) incremental exercise on a treadmill, c) indirext calorimetry at rest. $\dot{\mathrm{V}} \mathrm{O}_{2}$ : oxygen consumption. $\mathrm{RER}_{\text {peak }}$ : peak of respiratory exchange ratio $=\dot{\mathrm{V}} \mathrm{VO}_{2} / \mathrm{VO}_{2}$. $\mathrm{RER}_{\text {rest: }}:$ the lowest value of respiratory exchange ratio. Mean $\pm \mathrm{SD}$. Difference between T0 vs. T3: ${ }^{*} p<0.05,{ }^{* *} p<0.01,{ }^{* * *} p<0.01$. Training effect: difference between $\mathrm{C} v s$. E at T3: $\cdot p=0.08$, \#\# $p<$ 0.01 , \#\# $p<0.001$. Genotype effect: WT vs. KO. (With linear mixed-models approach with repeated measures ANOVA and simultaneous tests for general linear hypotheses).

exercise training we extended our analysis to various parameters, including $\mathrm{O}_{2}$ consumption, $\mathrm{CO}_{2}$ production, energy expenditure etc. (Table 2). The ratio of $\dot{\mathrm{V}} \mathrm{O}_{2 \text { peak }}$ to lean mass, indicative of specific muscle endurance adaptation, was significantly increased at T3 in exercised mice as compared to controls, in both WT and Srf KO mice. Some calorimetric parameters such as $\mathrm{RER}_{\text {peak }}$, representative of the maximum ratio between emitted $\mathrm{CO}_{2}$ and consumed $\mathrm{O}_{2}, \dot{\mathrm{V}} \mathrm{O}_{2}$ mean and $\dot{\mathrm{V}} \mathrm{CO}_{2}$ mean improved significantly between T0 and T3 in WT (E) mice: these parameters were the only ones for which WT differed from the Srf $\mathrm{KO}$ mice upon exercise (E groups).

WT and Srf KO mice did not display major differences in the willingness to perform exercise nor in the basal metabolic rate

Since the exercise training was based on voluntary exercise, which could result in a different amount of running performed between the group, it is worthwhile noting that both WT and KO groups show a similar running distance over the 3 -week protocol period with a regular running time (Table 2). These results not only indicate that the other outputs were not biased by different behaviors during the exercise training, but also represent a functional, behavioral essay per se, showing that overall WT and Srf KO mice have a similar attitude toward free exercise. To further compare the WT and Srf $\mathrm{KO}$ groups, we also performed calorimetric tests in the two groups at rest, i.e. not upon speed test. Overall, we noticed a significant effect of exercise on the basal metabolic rate at rest (Table 2, comparison of the $\mathrm{C}$ vs. $\mathrm{E}$ groups, shown in the $\mathrm{P}$ column). In addition, we noticed that over time (T3 vs T0) there was an increase in $\dot{\mathrm{V}} 2$ rest, in both WT and Srf KO mice, which only in the latter remains significant when normalized by the mean mass. No major differences were found among specific groups for what concerns RER $_{\text {rest }}$ (Table 2).

\section{Discussion}

Exercise effects on body composition and performance may depend on the genetic background. We hypothesized that lack of Srf in skeletal muscle would influence skeletal muscle adaptation to endurance exercise, given the pivotal role Srf plays in muscle tissues and the fact that Srf translates mechanical cues into genetic expression. ${ }^{15,34} \mathrm{Srf}$ is a transcriptional target of miR133a, whose levels are affected by exercise, ${ }^{35}$ and RhoSTARS signaling induced by exercise typically leads to Srf activation. ${ }^{36}$ However, very little is known on Srf and exercise: this is the first study describing in detail the physical adaptation to endurance exercise in the absence of muscle-specific Srf expression. Muscle mass strength displayed by Srf KO mice is not significantly different from their WT siblings at 8 weeks of age. Premature aging (sarcopenia) is observed in the musculature of mice lacking Srf expression. ${ }^{21}$ In particular, 13 months after 
the induction of Srf deletion, Srf mutant mice developed IIB myofiber-specific atrophy accompanied by a metabolic switch towards a more oxidative phenotype, muscular lipid accumulation, sarcomere disorganization and fibrosis. ${ }^{21}$ Our observations are important since they suggest that the modifications due to the absence of Srf take time to occur and that young, Srf KO mice can be compared to WT mice in the absence of major basal differences in muscle mass and function. KO and WT mice spend the same amount of time on the wheel and perform the same amount of exercise, suggesting that the genotype has no major effects on mouse physical activity as a behavior; this was not granted, since we had previously shown that even subtle genetic differences may result in differences in mouse physical activity. ${ }^{6,8}$ We performed an extensive analysis of both WT and Srf KO mice, by comparing: body composition (fat and lean body mass), muscle function (maximal and normalized strength), endurance adaptation (maximal speed, $\mathrm{O}_{2}$ consumption), and metabolism (RER, i.e. $\dot{\mathrm{V} C O} 2 / \dot{\mathrm{VO}}_{2}$ ratio, energy expenditure, substrate consumption for energy production). Taken together, our results showed that exercise affects Srf KO mice muscle mass and function and induces endurance fitness similarly to what observed in the WT population. The findings that mice of both genotypes maintain their total and fat mass and tend to gain lean mass are consistent with previous studies. ${ }^{37,38}$ Wild-type and Srf KO mice have approximately $2 \mathrm{~g}$ of fat, as expected for mice. ${ }^{39}$ The increase in lean mass in Srf KO mice could account for the significant increase in force displayed by these animals. The average daily distances run by WT and Srf KO mice in our study were $5.54 \mathrm{~km}$ and $5.68 \mathrm{~km}$ per day, respectively, within a previously reported range of 4.2-6.5 km/day. ${ }^{37,38}$ Trained WT and KO mice showed a significant improvement in maximum speed, a significant increase of $\dot{\mathrm{VO}}_{2}$ peak and a higher ratio of $\dot{\mathrm{VO}}_{2}$ peak to lean mass following exercise training compared to non-active mice. This result is in agreement with Lightfoot et al. who demonstrated that lineage variations in mice may influence responses in aerobic power. ${ }^{40}$ The adaptation to exercise may contribute to the muscle mass preservation and explain other observations, such as that WR during cisplatin treatment maintained lean body mass and muscle. ${ }^{41}$ This is particularly relevant in the light of the fact that cisplatin, as a chemotherapic agent, induces muscle wasting per se, suggesting the need of countermeasures. ${ }^{42,43} \mathrm{Srf}$ is a transcription factor of pivotal importance for muscle tissues and its genetic models are widely used to study its role in muscle homeostasis, physiology and pathology. We have compared mouse adaptation to endurance exercise, in the absence or presence of muscle-specific Srf expression. We found, as expected, a major improvement in the aerobic capacity and muscle function following exercise; however, no major differences were observed in Srf $\mathrm{KO}$ mice as compared to WT mice, following exercise. Our observations suggest that Srf is not required for an early (within 3 weeks) adaptation to spontaneous exercise and that Srf KO mice behave similarly to the WT in terms of spontaneous physical activity and the resulting adaptive responses. Taken into account the positive effects of exercise against cancer cachexia, it would be interesting whether exercise requires Srf to be effective in such context. Since Srf does not interfere with adaptation to exercise, skeletal muscle Srf KO mice can be used in functional muscle studies without the results being biased by the lack of Srf.

\section{List of acronyms}

Cre-LoxP - Cre-LoxP recombination

Mrtf-A - myocardin related transcription factor A

RER - respiratory exchange ratio

Smax - maximal running speed

Srf - serum response factor

Tam - tamoxifen

VO2 max - maximal oxygen consumption

\section{Authors contributions}

$\mathrm{HD}$ and $\mathrm{MH}$ performed the experiments and elaborated the data, ND had a major role in writing the manuscript, $\mathrm{ZL}$ and AS contributed to the interpretation of the data and to the manuscript preparation, PN and DC ideated and coordinated the experimental work, corrected the manuscript and provided financial support

\section{Acknowledgments}

We thank Pr Arnaud Ferry (INSERM U974, Institut de Myologie, CNRS FRE3617, UPMC, Université Paris Descartes, Sorbonne Paris Cité) for his outstanding technical support. We acknowledge the technical facility "Performance \& Metabolism in Mice" (PMM, IRMES, University Paris Descartes, Sorbonne Paris Cité - INSEP, Paris, France) for performance and metabolic analysis.

\section{Funding}

DC is funded by AFM (2017-20603), EFEM 2016, IBPS (2014), NIH (2013-1R01CA108857-01 subcontractor) and UPMC Emergence (2011-EME1115). DC and AS were funded by ANR (2013-J13R191). This research was also supported by labex GR-Ex. The labex GR-Ex, reference ANR-11-LABX-0051 is funded by the program "Investissements d'avenir" of the French National Research Agency, reference ANR-11-IDEX0005-02

\section{Conflict of Interest}

The authors declare no conflict of interest.

\section{Ethical Publication Statement}

We confirm that we have read the Journal's position on issues involved in ethical publication and affirm that this report is consistent with those guidelines. 


\section{Corresponding Author}

Philippe Noirez

Postal Adress: IRMES, EA7329, CUSP, 45 rue des St Pères, 75006 Paris, France

Phone number: +3314174 4129 and Fax number: +33 141744175

Email: philippe.noirez@parisdescartes.fr

\section{E-mails of co-authors}

Haidar Djemai: Haidar Djemai:

haidar.djemai@gmail.com

Medhi Hassani: medhihsn@gmail.com

Nissrine Daou: daou.nissrine@gmail.com

Zhenlin Li: zhenlin.li@upmc.fr

Athanassia Sotiropoulos:

athanassia.sotiropoulos@inserm.fr

Dario Coletti: dario.coletti@uniromal.it

\section{References}

1. Holloszy JO, Booth FW. Biochemical adaptations to endurance exercise in muscle. Annu Rev Physiol 1976;38:273-91.

2. Protasi F. Mitochondria Association to Calcium Release Units is Controlled by Age and Muscle Activity. Eur J Transl Myol 2015;25:257-62.

3. Zampieri S, Mosole S, Lofler S, et al. Physical Exercise in Aging: Nine Weeks of Leg Press or Electrical Stimulation Training in 70 Years Old Sedentary Elderly People. Eur J Transl Myol 2015;25:237-42.

4. Holloszy JO, Coyle EF. Adaptations of skeletal muscle to endurance exercise and their metabolic consequences. J Appl Physiol Respir Environ Exerc Physiol 1984;56:831-8.

5. Barone R, Macaluso F, Sangiorgi C, et al. Skeletal muscle Heat shock protein 60 increases after endurance training and induces peroxisome proliferator-activated receptor gamma coactivator 1 alpha1 expression. Sci Rep 2016;6:19781.

6. Coletti D, Adamo S, Moresi V. Of Faeces and Sweat. How Much a Mouse is Willing to Run: Having a Hard Time Measuring Spontaneous Physical Activity in Different Mouse Sub-Strains. Eur J Transl Myol 2017;27:6483.

7. Coletti D, Aulino P, Pigna E, et al. Spontaneous Physical Activity Downregulates Pax7 in Cancer Cachexia. Stem Cells Int 2016;2016:6729268.

8. Coletti D, Berardi E, Aulino P, et al. Substrains of inbred mice differ in their physical activity as a behavior. Scientific World Journal 2013;2013:237260.

9. Merico A, Cavinato M, Gregorio C, et al. Effects of combined endurance and resistance training in Amyotrophic Lateral Sclerosis: A pilot, randomized, controlled study. Eur J Transl Myol 2018;28:7278.

10. Aline G, Sotiropoulos A. Srf: A key factor controlling skeletal muscle hypertrophy by enhancing the recruitment of muscle stem cells. Bioarchitecture 2012;2:88-90.

11. Coletti D, Daou N, Hassani M, et al. Serum Response Factor in Muscle Tissues: From Development to Ageing. Eur J Transl Myol 2016;26:6008.

12. Posern G, Treisman R. Actin' together: serum response factor, its cofactors and the link to signal transduction. Trends Cell Biol 2006;16:588-96.

13. Pipes GC, Creemers EE, Olson EN. The myocardin family of transcriptional coactivators: versatile regulators of cell growth, migration, and myogenesis. Genes Dev 2006;20:1545-56.

14. Miralles F, Posern G, Zaromytidou AI, et al. Actin dynamics control SRF activity by regulation of its coactivator MAL. Cell 2003;113:329-42.

15. Sotiropoulos A, Gineitis D, Copeland J, et al. Signal-regulated activation of serum response factor is mediated by changes in actin dynamics. Cell 1999;98:159-69.

16. Olson EN, Nordheim A. Linking actin dynamics and gene transcription to drive cellular motile functions. Nat Rev Mol Cell Biol 2010;11:353-65.

17. Mendez MG, Janmey PA. Transcription factor regulation by mechanical stress. Int J Biochem Cell Biol 2012;44:728-32.

18. Charvet $\mathrm{C}$, Houbron C, Parlakian A, et al. New role for serum response factor in postnatal skeletal muscle growth and regeneration via the interleukin 4 and insulin-like growth factor 1 pathways. Mol Cell Biol 2006;26:6664-74.

19. Li S, Czubryt MP, McAnally J, et al. Requirement for serum response factor for skeletal muscle growth and maturation revealed by tissue-specific gene deletion in mice. Proc Natl Acad Sci U S A 2005;102:1082-7.

20. Guerci A, Lahoute C, Hebrard S, et al. Srfdependent paracrine signals produced by myofibers control satellite cell-mediated skeletal muscle hypertrophy. Cell Metab 2012;15:25-37.

21. Lahoute C, Sotiropoulos A, Favier M, et al. Premature aging in skeletal muscle lacking serum response factor. PLoS One 2008;3:e3910.

22. Carson JA, Wei L. Integrin signaling's potential for mediating gene expression in hypertrophying skeletal muscle. J Appl Physiol (1985) 2000;88:337-43.

23. Galmiche G, Labat C, Mericskay M, et al. Inactivation of serum response factor contributes to decrease vascular muscular tone and arterial stiffness in mice. Circ Res 2013;112:1035-45.

24. Parlakian A, Charvet C, Escoubet B, et al. Temporally controlled onset of dilated cardiomyopathy through disruption of the SRF gene in adult heart. Circulation 2005;112:2930-9.

25. Collard L, Herledan G, Pincini A, et al. Nuclear actin and myocardin-related transcription factors 
control disuse muscle atrophy through regulation of Srf activity. J Cell Sci 2014;127:5157-63.

26. Schuler M, Ali F, Metzger E, et al. Temporally controlled targeted somatic mutagenesis in skeletal muscles of the mouse. Genesis 2005;41:165-70.

27. Parlakian A, Tuil D, Hamard G, et al. Targeted inactivation of serum response factor in the developing heart results in myocardial defects and embryonic lethality. Mol Cell Biol 2004;24:5281-9.

28. Deloux R, Vitiello D, Mougenot N, et al. Voluntary Exercise Improves Cardiac Function and Prevents Cardiac Remodeling in a Mouse Model of Dilated Cardiomyopathy. Front Physiol 2017;8:899.

29. Lemaire KK, Thomasson R, Noirez P, et al. Commentary: Validation of a Ramp Running Protocol for Determination of the True VO2max in Mice. Front Physiol 2017;8:330.

30. Bruss MD, Khambatta CF, Ruby MA, et al. Calorie restriction increases fatty acid synthesis and whole body fat oxidation rates. Am J Physiol Endocrinol Metab 2010;298:E108-16.

31. Molinero A, Fernandez-Perez A, Mogas A, et al. Role of muscle IL-6 in gender-specific metabolism in mice. PLoS One 2017;12:e0173675.

32. Weir JB. New methods for calculating metabolic rate with special reference to protein metabolism. J Physiol 1949;109:1-9.

33. Frayn KN. Calculation of substrate oxidation rates in vivo from gaseous exchange. J Appl Physiol Respir Environ Exerc Physiol 1983;55:628-34.

34. Posern G, Sotiropoulos A, Treisman R. Mutant actins demonstrate a role for unpolymerized actin in control of transcription by serum response factor. Mol Biol Cell 2002;13:4167-78.

35. Ramos AE, Lo C, Estephan L, et al. Specific circulating microRNAs display dose-dependent responses to variable intensity and duration of endurance exercise. Am J Physiol Heart Circ Physiol 2018.
36. Wallace MA, Hock MB, Hazen BC, et al. Striated muscle activator of Rho signalling (STARS) is a PGC-1alpha/oestrogen-related receptor-alpha target gene and is upregulated in human skeletal muscle after endurance exercise. J Physiol 2011;589:2027-39.

37. Rogers CJ, Berrigan D, Zaharoff DA, et al. Energy restriction and exercise differentially enhance components of systemic and mucosal immunity in mice. J Nutr 2008;138:115-22.

38. Hiramatsu L, Garland T, Jr. Mice selectively bred for high voluntary wheel-running behavior conserve more fat despite increased exercise. Physiol Behav 2018;194:1-8.

39. Hiramatsu L, Kay JC, Thompson Z, et al. Maternal exposure to Western diet affects adult body composition and voluntary wheel running in a genotype-specific manner in mice. Physiol Behav 2017;179:235-45.

40. Lightfoot JT, Leamy L, Pomp D, et al. Strain screen and haplotype association mapping of wheel running in inbred mouse strains. J Appl Physiol (1985) 2010;109:623-34.

41. Hojman P, Fjelbye J, Zerahn B, et al. Voluntary exercise prevents cisplatin-induced muscle wasting during chemotherapy in mice. PLoS One 2014;9:e109030.

42. Coletti D. Chemotherapy-induced muscle wasting: an update. Eur J Transl Myol 2018;28:7587.

43. Damrauer JS, Stadler ME, Acharyya S, et al. Chemotherapy-induced muscle wasting: association with NF-kappaB and cancer cachexia. Eur J Transl Myol 2018;28:7590.

Submissions: $\quad$ April, 05, 2019

Revision received: May 03, 2019

Acceptance: $\quad$ May 03, 2019 